\title{
Tapped Granular Packings Described as Complex Networks
}

\author{
Roberto Arévalo ${ }^{a}$, \\ Luis A. Pugnalonib, \\ Diego Maza ${ }^{\mathrm{c}}$ \\ Iker Zuriguel ${ }^{\mathrm{c}}$ a \\ CNR-SPIN, Dipartimento di Scienze Fisiche,Universitá di Napoli "Federico II", Naples, Italy. \\ Departamento de Ingeniería Mecánica, Facultad Regional La Plata, \\ Universidad Tecnológica Nacional, Av. 60 esq. 124 s/n, 1900 La Plata, Argentina. \\ Departamento de Física, Facultad de Ciencias, \\ Universidad de Navarra, E-31080 Pamplona, Spain.
}

December 18, 2013

\begin{abstract}
We characterize the structure of simulated two-dimensional granular packings using concepts from complex networks theory. The packings are generated by a simulated tapping protocol, which allows us to obtain states in mechanical equilibrium in a wide range of densities. We show that our characterization method is able to discriminate non-equivalent states that have the same density. We do this by examining differences in the topological structure of the contact network of the packings. In particular, we find that the polygons of the network are specially sensitive probes for the contact structure. Additionally, we compare the network properties obtained in two different scenarios: the tapped and a compressed system.
\end{abstract}

\section{Introduction}

The study of packings has enjoyed a long history both in physics and mathematics [1]. However, most of the interest has been traditionally centered around ordered structures such as stacks of cannonballs and the perfection of the honeycomb. Disordered packings have atracted real interest only rather recently. One can consider that this starts with the work of Bernal [2], who developed a model for the structure of fluids by considering a random packing of spheres, having a density of around 0.64. This density has been found to be the highest attainable in a random packing by a number of experimental techniques and computer simulations [3], thus making the Bernal packing a well defined 
bound (known as random close packing), at least empirically. The debate of the fundamental significance of this density is still very alive.

In the present work, we study a two-dimensional analog of the Bernal packing, i.e., disordered packings of disks in mechanical equilibrium. We focus on the properties of steady state configurations generated by a simulated tapping protocol: a bed of grains inside a container which is subjected to vertical pulsed agitation, allowing the grains to relax between successive excitations. By tuning the dimensionless vertical acceleration $\Gamma$ of the shaking, we can explore different states of mechanical equilibrium. This situation has been widely studied; experimentally $[4,5,6,7,9,10]$, by means of simulations $[8,9,10,11]$, and theoretically [12].

The system we propose to study plays a central role in the theoretical approach to granular media. The packings obtained are reacheable from one another [9], and thus fall within the scope of Edwards' theory of granular materials in mechanical equilibrium. This theory was first proposed by Edwards and Oakeshott [12] in formal analogy with the statistical theory that underlies thermodynamics. Initially, Edwards' theory was developed using only the number of grains $N$ and the volume $V$ of the pack (or equivalently, the packing fraction $\phi$ ) to characterize the macroscopic states. However, it has been recently suggested that the so called $N V$ ensemble should be extended, and take into account the stress propagation in the system to achieve a complete description of a granular packing $[13,14,15,16,17,18]$.

Recent experiments and simulations $[9,10,19]$ have shown that, when a layer of grains is tapped, the steady state packing fraction $\phi$ is not a monotonic function of the excitation intensity. Instead, a minimum packing fraction $\phi_{\min }$ is achieved at a certain intensity $\Gamma_{\min }$. Beyond this value, the packing fraction increases again. This non-monotonic behavior implies that steady states with equal average packing fractions can be reached with very different tap intensities. Then, the question of whether these states are statistically equivalent or not, naturally arises. This question has been thoroughly studied by means of simulations in Refs. $[9,10]$ where it is found that the force moment tensor $\Sigma$ increases monotonically with $\Gamma$. Hence, it is concluded that states at both sides of the minimum are not equivalent, and that $\Sigma$ is a suitable candidate to complete the variables that describe a granular system at equilibrium at the macroscopic level.

Provided that $\Sigma$ is revealed to be able to distinguish steady states of equal $\phi$, one would like to explore what other descriptors would be also valid to differentiate configurations which are not discernible by $\phi$. Since the external stress applied to the system is equilibrated exclusively by contacts, it seems reasonable to think in the properties of the contact network. We can then use the concepts and tools of complex networks [20] to analyze the properties of the contact structure. These will be used to evaluate whether there is any network property that allows a clear distinction between states with the same $\phi$. At this point, let us note that any static granular system can be considered in terms of nodes (the grains) and edges (the contacts between grains). This approach has been recently applied to address a wide variety of granular phenomena such as 
porosity [21], force distribution [22], rheology [23], signal propagation [24] and jamming transition $[25,26]$.

The aim of the present work is to characterize the contact networks of the different steady states of packings generated by tapping. We will demonstrate that states with the same volume obtained with different tap intensities exhibit discernible contact structures, rendering them non-equivalent.

\section{Simulation and tapping protocol}

We use soft-particle molecular dynamics simulations in $2 D$, in which static friction is implemented through the usual Cundall-Strack model [27]. The details of the implementation have been described elsewhere [28], and in the following we give the values of the interaction parameters used in the present work. The friction coefficient is $\mu=0.5$, stiffness in the normal direction of the contact $k_{n}=10^{5}(\mathrm{mg} / \mathrm{d})$, damping parameter in the normal direction $\gamma_{n}=300(\mathrm{mg} / \mathrm{d})$, stiffness in the tangential direction $k_{s}=\frac{2}{7} k_{n}$ and the damping parameter in the tangential direction $\gamma_{s}=200(\mathrm{mg} / \mathrm{d})$. The integration time step is set to $\delta=10^{-4} \sqrt{d / g}$. The confining box (13.39d-wide and infinitely high) contains $N=512$ monosized disks. We choose a box size which is inconmensurate with the disc size to reduce crystallization. At the same time, we find that this measure prevents the formation of a regular network of contacts, in spite of the grains being monosized. Units are reduced with the diameter of the disks, $d$, the disk mass, $m$, and the acceleration of gravity, $g$.

Tapping is simulated by moving the confining box in the vertical direction following a half sine wave trajectory $[A \sin (2 \pi \nu t)(1-\Theta(2 \pi \nu t-\pi))]$. The excitation is applied with a constant frequency $\nu=\pi / 2(g / d)^{1 / 2}$, and hence it is solely controlled through the amplitude, $A$. The tap is characterized by the dimensionless parameter $\Gamma=A(2 \pi \nu)^{2} / g$ although we would like to note that other parameters (like the adimensionless energy supplied to the system [29]) have been reported to be more suitable. In order to decide when the system has reached mechanical equilibrium, we implement a robust criterion based on the stability of particle contacts [28]. Then, the packing fraction is calculated and a new tap is applied to the sample. Packing fraction was calculated in a slab of the bed that covers $50 \%$ of the height of the column and is centered with the center of mass of the system. Averages were taken over 500 taps (configurations) after we ensure that the system has reached a steady-state for each value of $\Gamma$. In particular, we discard the 500 initial configurations in order to avoid any transient. By assuming that the values for the packing fraction obtained at each tap are normally distributed, we estimate the uncertainty of the mean packing fraction by calculating the upper and lower limits for a $95 \%$ confidence interval (we will apply the same criterion for all the magnitudes introduced in the next sections). Using this protocol, we obtain the curve of mean packing fraction $\phi$ in the steady state as a function of $\Gamma$ (Fig. 1). At low values of $\Gamma$, increasing the intensity of the taps leads to a decrease in the packing fraction. Then, beyond a certain value $\Gamma_{\min }$, this tendency is reversed and the density 
increases again up to values slightly below the ones obtained at low excitations. This qualitative behavior is independent of the frequency $\nu$ of the taps, although the position of the minimum is shifted to higher values of $\Gamma$ when $\nu$ is increased [10].

\section{Structure of the contact network}

We now proceed to analyze the structure of the contact network of the tapped packings. It will be shown that the topology of the contacts allows to distinguish states with the same packing fraction at both sides of the minimum without computing the forces.

The network is readily defined by taking every grain as a node and every contact as an edge [Fig. 2]. Note that, due to the gravity, in our samples there are no rattlers, i.e. particles without any contact. Thus, there is no ambiguity as to whether or not a grain participates in stress transmission. Once the graph is defined, the following properties [30] are computed: (a) the mean shortest distance (or mean topological distance) $\langle l\rangle$ between two given nodes measured in number of edges; (b) the diameter $D$ of the network, which is the largest topological distance in the graph; and (c) the degree of the network $\langle k\rangle$ which is equivalent to the average contact number per grain $Z$ used in the granular community. In addition, we measure the populations of polygons. These are closed loops of contacts around an empty void, and their size is measured in number of edges. In the following, we term these empty closed loops simply as triangles, squares, pentagons and so on. Polygons similar to the ones considered in this work were introduced in [31] where it was shown that odd loops of contacts are responsible for the rigidity of granular samples due to the frustration of rotations they introduce in the system.

In Figs. 3(a)-(c) we report the behavior of $\langle l\rangle, D$ and $\langle k\rangle$ as functions of the tapping intensity, $\Gamma$. The shape of the curves correlates with that of the packing fraction in Fig. 1. At low tapping intensities both $\langle l\rangle$ and $D$ increase with $\Gamma$, while $\langle k\rangle$ decreases. This corresponds to a process of disconnection of the network in which links are removed to allow for an increase of porosity. Topologically, the paths among nodes are modified in a way such that grains become more separated. Trends are reversed beyond a point around $\Gamma \simeq 4.0$, which corresponds to the minimum packing fraction. From this point the network is increasingly connected as revealed by the increase of $\langle k\rangle$. As a result, the distances between grains and the average diameter of the network tend to values similar to those obtained for low tapping intensities.

In Figs. 3(d)-(f) the values of $\langle l\rangle, D$ and $\langle k\rangle$ are reported as functions of the packing fraction $\phi$. These plots show the existence of two separated branches, one before the minimum and another after this point. Hence, the topology of the contact network is actually different at both sides of the minimum density, and these descriptors allow to discriminate different mechanical equilibrium states that have the same packing fraction. Note however that this distinction relies on a high accuracy of the data, since the relative differences in $\langle l\rangle, D$ and $\langle k\rangle$ 
between states with the same $\phi$ are very small.

The populations of contact loops (polygons) of the network with respect to $\Gamma$ are shown in left column of Fig. 4. According to the data, the polygons may be classified into two categories: small polygons, comprised by triangles and squares; and big polygons, comprised by pentagons and heptagons (see below for the case of hexagons). For low tapping intensities, the number of small polygons decreases upon increasing $\Gamma$, while the number of big polygons increases. Beyond the point of minimum packing fraction, the trends are reversed, small polygons increase their populations with $\Gamma$, while the number of big polygons is reduced. Hexagons do not neatly fit in either category due to its monotonic behavior with respect to $\Gamma$. This is a unique feature which has not been observed in any other descriptor -neither geometrical nor topological- considered so far. We speculate that this particular behavior of the number of hexagons is strongly related with the boundary effects. Most hexagons observed are located near the vertical walls of the container (as exemplified by Fig. 2). This phenomenon could be associated with the special columnar arrangements of particles induced by the walls $[32,33,34]$.

The behavior of the number of polygons within the network can be easily linked with the results obtained for the other topological properties. Indeed, increasing the number of small polygons adds connectivity to the network. For example, if we consider a group of three nodes connected through two edges, the distance is $\langle l\rangle=2$ and the average connectivity is $\langle k\rangle=4 / 3$. If a triangle is formed adding an extra edge, the distance reduces to $\langle l\rangle=1$ and the connectivity rises to $\langle k\rangle=2$. For $\Gamma<\Gamma_{\min }$, increasing $\Gamma$ leads to a reduction in the number of small polygons and, hence, the network connectivity is reduced. For $\Gamma>\Gamma_{\min }$, the behavior reverses with large polygons being divided into smaller ones by new edges.

The population of polygons as a function of the density (see Figs 4(f)-(j)) reveals - as the other topological descriptors in Fig. 3- the existence of two branches, one at each side of the minimum. This behavior is specially clear for the case of triangles and hexagons. In brief, we can state that the branch of higher $\Gamma$ is characterized by a higher number of triangles and a lower number of hexagons, in comparison with the branch of lower $\Gamma$. Let us stress that the relative differences between the two branches obtained for the number of triangles and hexagons are quite important: $50 \%$ in the best case. This result notably differs from the small differences between the two branches obtained for $\langle l\rangle, D$ and $\langle k\rangle$. Hence, it can be stated that the population of polygons is a more suitable parameter to distinguish between states with the same $\phi$ than other topological descriptors. 


\section{Force networks. Relation to compressed sys- tems}

Once the topological structure of the contact network has been analyzed, it seems interesting to define more general force networks. Those will be used to compare tapped systems with compressed ones. To this end, let us introduce the parameter $f^{*}=F_{t h} /\langle F\rangle$, where $\langle F\rangle$ is the average normal contact force and $F_{t h}$ is a threshold force. A force network is defined by considering as edges only the contacts in which the normal force is larger than $F_{t h}$. Thus, for $f^{*}=0$ the force network is identical to the contact network, while for larger values of $f^{*}$ diluted graphs with decreasing number of conections and nodes are obtained. Indeed, when the parameter $f^{*}$ is increased, the network disaggregates giving rise to the formation of clusters: groups of nodes which are mutually linked but disconnected from other groups, see Fig. 2. The giant component $(G C)$ of the network is defined as the cluster with largest size, measured in number of nodes.

Due to gravity, in the tapped packings there is a gradient of forces in the vertical direction, with large forces being more common close to the base of the layer, and weaker contacts concentrated close to the free surface. To avoid that this spatial inhomogeneity in the force distribution affects our conclusions, the results concerning forces presented in this and the next section, are obtained measuring in a narrow slab of height $10 d$ centered at a distance $10 d$ from the base.

Using this definition, the topological properties of the networks can be studied as functions of the force threshold [22]. In Fig. 5 a comparison is made between an isotropically compressed system that is jammed [25] (left column) and the tapped samples studied in this work (right column). From the data for the topological distance and the giant component, a different qualitative behavior is revealed for both types of networks. The compressed system shows a sharp transition for a force approximately equal to the average, $f^{*} \simeq 1$. For $f^{*} \geq 1$ the topological distance and the giant component are essentially zero, while, for $f^{*} \leq 1$ they jump to values close to those corresponding to the contact network. This transition takes place in a very narrow range of $f^{*}$. In contrast, the corresponding magnitudes in tapped systems behave smoothly in all the range of $f^{*}$ values. The giant component grows monotonically when $f^{*}$ is reduced, and shows no plateau at small forces. The topological distance shows a peak around $f^{*} \simeq 1.5$ for low densities, and another at $f^{*} \simeq 1$ for the highest. However, the behavior does not correspond to a sharp transition as in the case of jammed compressed systems. The same pattern repeats in the results for the population of polygons. In the case of compressed networks, all the populations decay exponentially for $f^{*} \geq 1$, while their behavior is diverse for small values of the parameter. The tapped networks show a monotonic decay in the populations of all the polygons. The results presented in Fig. 5(f) correspond to a packing fraction $\phi=0.84$ but similar results are obtained for the other packing fractions analysed. 


\section{Contact forces}

An additional hint to the distinct behavior found in tapping and compression may be obtained from the distribution of normal contact forces. In Fig. 6a the normalized distribution of contact forces is shown for the compressed system in two different situations. For $\phi=0.82$ the system is not jammed as it is below the critical value of $\phi$, and the distribution of normal forces decreases monotonically. On the contrary, for $\phi=0.85$-when the system is jammed- the distribution shows a maximum around a force equal to the average. In Fig. $6 \mathrm{~b}$ the corresponding force distributions are presented for tapped systems. Interestingly, the curves are monotonous for every packing density. The emergence of a peak in the force distribution was recognized in [35] as a signature of the development of a yield stress and, hence, the transition to a jammed state. According to this, the tapped samples are not jammed: they would deform by the application of a shear stress. Note that this difference in the internal state of the forces is also reflected in the topological properties of the diluted graphs displayed in section 4 .

Another suggestive result observed from Fig. $6 \mathrm{~b}$ is that the histograms for the lowest densities show a fairly exponential decay. By contrast, an enhancement of large forces is observed for high densities. This enhancement is observed for packings obtained both at low and high $\Gamma$.

Let us now further analyze the information on the contact forces of the mechanical equilibrium states obtained by tapping. In Fig. 7 we show the average value of the normal force in each type of polygon and for every value of $\phi$. The general trend is that the force increases with the number of sides of the polygon. The fact that small polygons in the network allocate smaller forces than big polygons was already observed for the compressed system [25, 26].

An intuitive explanation for the presence of small forces in the small polygons can be borrowed from Radjai et al. [36] who proposed that the network of contacts in a granular packing is composed of two subnetworks: a weak one formed by links carrying forces smaller than the average and another strong constituted by links carrying forces above the average. Our work suggests that, for the two scenarios analyzed, the filamentary structure mainly carries large forces whereas the polygonal structure allocates most of the weak forces.

In granular systems, the occurrence of force chains is related to the ability of a granular sample to resist the external loads. However, it has been pointed out that an additional ingredient is needed, given that a chain of disks or spheres is prone to buckle under longitudinal load. It has been suggested [37, 25] that the required stability can be provided by the triangles of the network. Effectively, triangles are natural units of rigidity due to the frustration of rotations and their minimal character (smallest loops). 


\section{Conclusions}

We have studied the contact network structure of the steady states reached by a granular layer when submitted to vertical tapping. It has been recently shown [9] that the volume fraction of the system is not a monotonic function of the tap amplitude and that an extra variable is necessary to univocally identify each steady state of the system [10].

In this work, we have implemented a series of topological measurements based on the contact network of the resulting packings. This approach allows us to distinguish between states of equal packing fraction that are known to differ in the average moment tensor. The suitability of a topological approach seems obvious as the interparticle contacts univocally determine the stress transmission of each mechanical equilibrium state. In this sense, the density or the number of nearest neighbors (defined by proximity rather than contact) seems a less precise parameter which is unable to detect small changes in the sample. The contact network, instead, will reflect such small changes, thus becoming a more suitable parameter to reflect the state of the system.

Among all the topological properties studied, we have found that the family of polygonal structures is the most sensitive to differentiate between states with the same packing fraction. We speculate that the mesoscopic nature of poly-

gons (they are based on contacts but also extend and consider the neighbors of neighbors) allows them to capture information lost in a purely local context.

The analysis of the diluted graphs obtained for different force thresholds puts in evidence that the topology of the force networks is sensitive to the internal state of the force distribution. The structure of the networks for compressed, jammed systems, in which a yield stress has developed, shows sharp features that are absent from the topological structure of the tapped systems. This result is corroborated by the study of the force distribution function in both cases. For the jammed compressed systems a peak is observed in the distribution signaling the development of yield stress. This peak is not observed, neither in the nonjammed compressed systems, nor in the tapped packings.

We also show that the normal force averaged over all the contacts of a polygon, increases with the number of their nodes. Attributing this behavior to the different roles of the polygons in regard to rigidity presents itself as a natural possibility. Effectively, triangles are minimal structures of rigidity due to the frustration of rotations. In this sense, our result is in accord with the idea of Tordesillas et al. [37] who claim that these topological structures provide a dual resistance to force chain buckling both by providing strong lateral support to the force chains and by impeding rotation of the particles.

Finally, let us remark the connection of polygons with the packing fraction in the system. Effectively, the natural role of polygons with more than three sides is the 'regulation' of packing fraction as, unlike triangles, these polygons enclose a void space. This is consistent with the decrease of the number of triangles and the increase of pentagons and heptagons when the packing fraction decreases. The decrease of squares and hexagons can be understood as a trade to increase the population of the odd-sided large polygons. 


\section{Acknowledgments}

R.A. thanks MIUR-FIRB RBFR081IUK for financial support. I.Z. and D.M. thank projects FIS2011-26675 (Spanish Government) and PIUNA (Universidad de Navarra).

\section{References}

[1] T. Aste and D. Weaire, The pursuit of perfect packing, Taylor \& Francis, London, 2008.

[2] J. D. Bernal, Nature 183 (1959) p.141.

[3] R. D. Kamien and A. J. Liu, Phys. Rev. Lett. 99, (2007) p.155501.

[4] E. R. Nowak, J. B. Knight, E. Ben-Naim, H. M. Jaeger, and S. R. Nagel, Phys. Rev. E 57 (1998) p.1971.

[5] E. R. Nowak, J. B. Knight, M. L. Povinelli, H. M. Jaeger, and S. R. Nagel, Powder Technol. 94 (1997) p.79.

[6] Ph. Ribière, P. Richard, P. Philippe, D. Bideau, and R. Delannay, Eur. Phys. J. E 22, (2007) p.249.

[7] M. Schröter, D. I. Goldman, and H. L. Swinney, Phys. Rev. E 71 (2005) p.030301(R).

[8] R. Arévalo, L. A. Pugnaloni, I. Zuriguel, and D. Maza, Phys. Rev. E 87 (2013) p.022203.

[9] L. A. Pugnaloni, I. Sánchez, P. A. Gago, J. Damas, I. Zuriguel, and D. Maza, Phys. Rev. E 82 (2010) p.050301(R).

[10] L. A. Pugnaloni, J. Damas, I. Zuriguel, and D. Maza, Papers in Physics 3, (2011) p.030004.

[11] M. Pica Ciamarra, A. Coniglio, and M. Nicodemi, Phys. Rev. Lett. 97, (2006) p.158001.

[12] S. F. Edwards and R. B. S. Oakeshott, Physica A 157 (1989) p.1080.

[13] J. H. Snoeijer, T. J. H Vlugt, W. G. Ellenbroek, M. van Hecke, J. M. J. van Leeuwen, Phys. Rev. E 70 (2004) p.061306.

[14] S. F. Edwards, Physica A 353 (2005) p.114.

[15] S. Henkes, C. S. OHern, B. Chakraborty, Phys. Rev. Lett. 99 (2007) p.038002.

[16] S. Henkes, B. Chakraborty, Phys. Rev. E. 79 (2009) p.061301. 
[17] R. Blumenfeld, S. F. Edwards, J. Phys. Chem. B 113 (2009) p.3981.

[18] B P Tighe, A R T van Eerd, T J H Vlugt, Phys. Rev. Lett. 100 (2008) p.238001.

[19] P. A. Gago, N. E. Bueno, and L. A. Pugnaloni, Granular Matter 11 (2009) p.365.

[20] M. E. J. Newman, SIAM Review 45, (2003) p.167.

[21] J. A. Dodds, J. Colloid. Interphase Sci. 77 (1980) p.317.

[22] S. Ostojic, E. Somfai, B. Nienhuis, Nature 439 (2006) p.828.

[23] D. M. Walker and A. Tordesillas, Phys. Rev. E 85 (2012) p.011304.

[24] D. S. Bassett, E. T. Owens, K. E. Daniels, M. A. Porter, Phys. Rev. E (in press).

[25] R. Arévalo, I. Zuriguel, and D. Maza, Phys. Rev. E 81 (2010) p.041302.

[26] R. Arévalo, I. Zuriguel, S. Ardanza-Trevijano, and D. Maza, Int. J. Of Bif. \& Chaos 20 (2010) p.897.

[27] P. A. Cundall, O. D. L. Strack, Geotechnique 29 (1979) p.47.

[28] R. Arévalo, D. Maza, and L. A. Pugnaloni, Phys. Rev. E 74 (2006) p.021303.

[29] F. Ludewig, S. Dorbolo, T. Gilet and N. Vandewalle, EPL 84 (2008) p.44001.

[30] R. Arévalo, I. Zuriguel and D. Maza, Int. J. Of Bif. \& Chaos 19 (2009) p.695.

[31] N. Rivier, J. Non-Cryst. Solids 352 (2006) p.42.

[32] I. Bartos and I. M. Janosi, Granular Matter 9 (2007) p.81.

[33] I. Zuriguel, T. Mullin, and R. Arévalo, Phys. Rev. E 77 (2008) p.061307.

[34] H. K. Chan, Phys. Rev. E. 84 (2011) p.050302(R).

[35] C. S. O'hern, S. A. Langer, A. J. Liu, S. R. Nagel, Phys. Rev. Lett. 86 (2001) p.111.

[36] F. Radjai, M. Jean, J. J. Moreau, and S. Roux, Phys. Rev. Lett. 77, (1996) 274.

[37] A. Tordesillas, D. M. Walker, and Q. Lin, Phys. Rev. E 81 (2010) p.011302. 
1. Mean packing fraction $\phi$ of the steady states as a function of the tap intensity $\Gamma$. The error bars correspond to the standard error estimated as the standard deviation divided by the square root of the number of configurations averaged (i.e., $\sqrt{500}$ ). The size of the error bars is similar to that of the symbols.

2. Part of one of the deposits analyzed. The left panel shows the contact network, the right panel the force network for $f^{*}=0.4$. In dark grey, edges joining the centers of grains in contact. The width of the edges is proportional to the corresponding normal force. Different loops (triangles, squares, pentagons and hexagons) can be observed.

3. Average shortest distance $\langle l\rangle$, average diameter $D$ and average connectivity $\langle k\rangle$ as functions of the tap intensity $\Gamma$ (a)-(c), and as functions of the mean packing fraction $\phi(\mathrm{d})-(\mathrm{f})$. The arrows indicate the direction of increasing $\Gamma$.

4. The populations of polygons defined in the text as a function of the tap intensity (a)-(e), and as a function of the mean packing fraction (f)-(j). The arrows indicate the direction of increasing $\Gamma$.

5. Average topological distance $\langle l\rangle$, giant component $C G$ and populations of polygons for compressed, jammed networks at $\phi=0.85$ (left) and tapped networks (right).

6. Normalized histogram of the normal contact forces for all the contacts. The variable $\left.f_{n}=F_{n} /<F_{n}\right\rangle$ is the normal contact force normalized by the average. a) Results for a compressed system before $(\phi=0.82)$ and after jamming $(\phi=0.85)$. b) Results for the packing states reached by tapping. Note that the values of packing fraction are shown in the caption in order of increasing $\Gamma$.

7. Normal force averaged over all the contacts of the polygons versus the size of the polygons. As in previous figures, the values of packing fraction are shown in the caption in order of increasing $\Gamma$. 


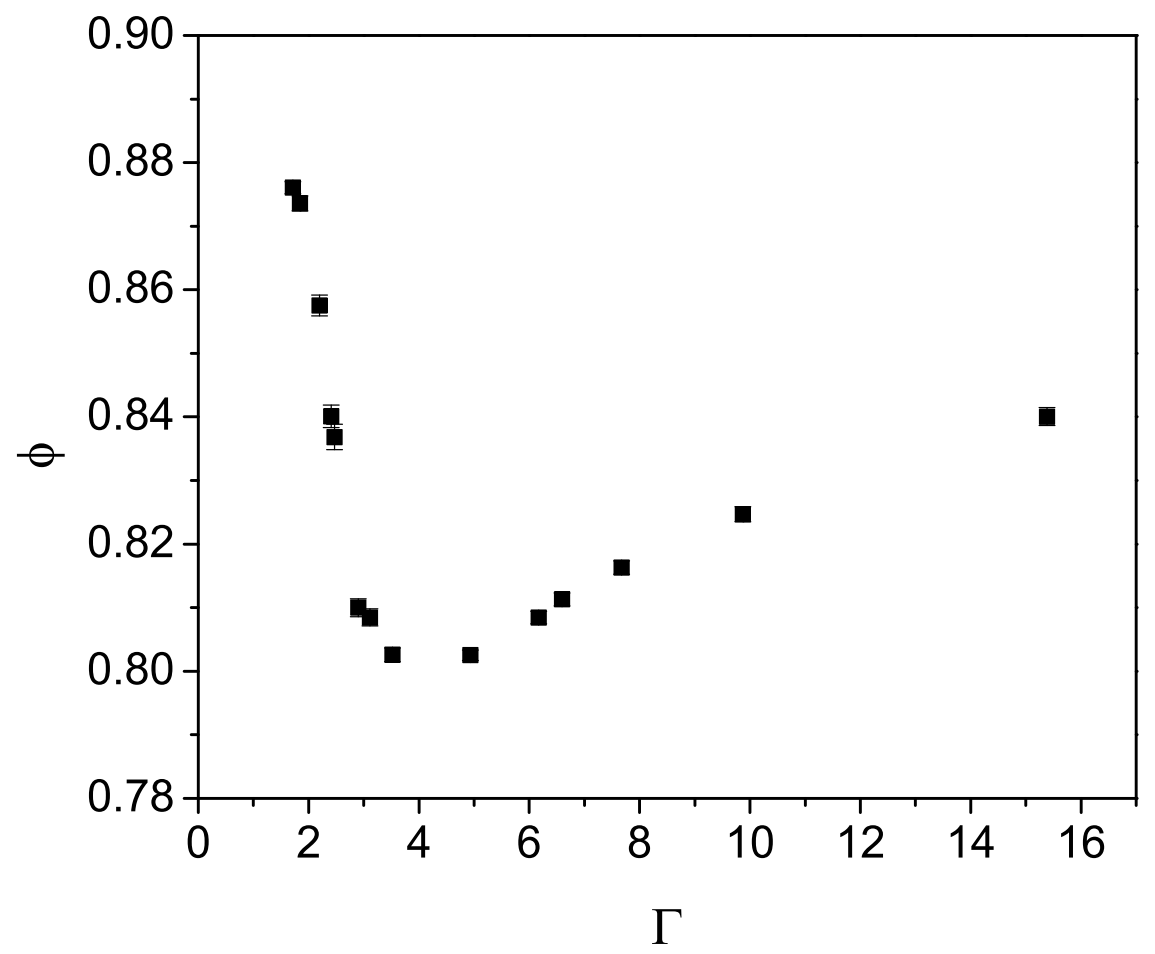

Figure 1: Mean packing fraction $\phi$ of the steady states as a function of the tap intensity $\Gamma$. The error bars correspond to the standard error estimated as the standard deviation divided by the square root of the number of configurations averaged (i.e., $\sqrt{500}$ ). The size of the error bars is similar to that of the symbols. 

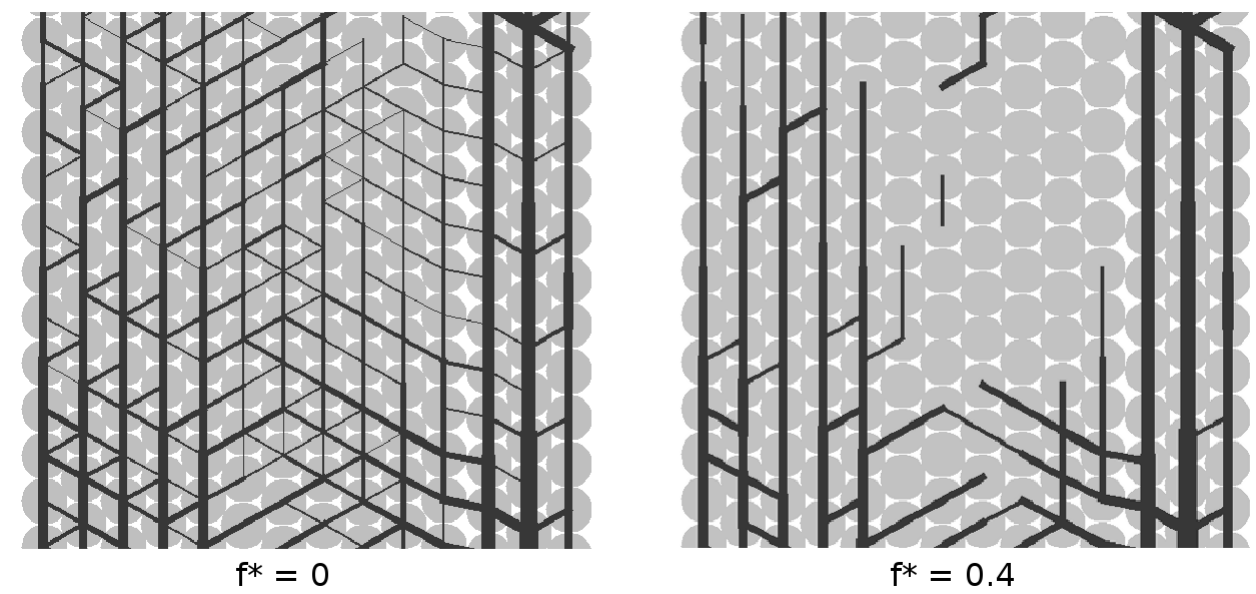

Figure 2: Part of one of the deposits analyzed. The left panel shows the contact network, the right panel the force network for $f^{*}=0.4$. In dark grey, edges joining the centers of grains in contact. The width of the edges is proportional to the corresponding normal force. Different loops (triangles, squares, pentagons and hexagons) can be observed. 

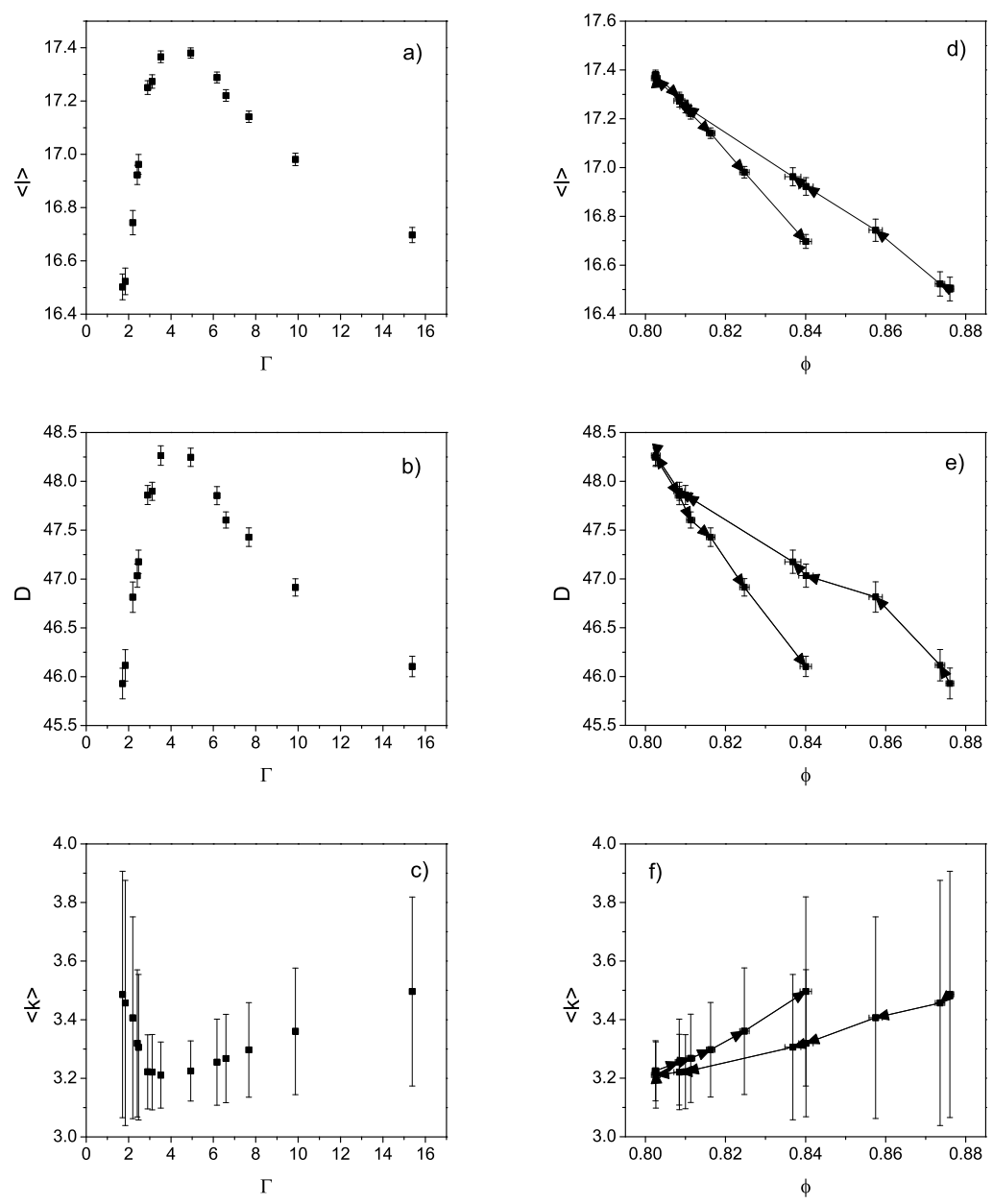

Figure 3: Average shortest distance $\langle l\rangle$, average diameter $D$ and average connectivity $\langle k\rangle$ as functions of the tap intensity $\Gamma$ (a)-(c), and as functions of the mean packing fraction $\phi(\mathrm{d})-(\mathrm{f})$. The arrows indicate the direction of increasing $\Gamma$. 

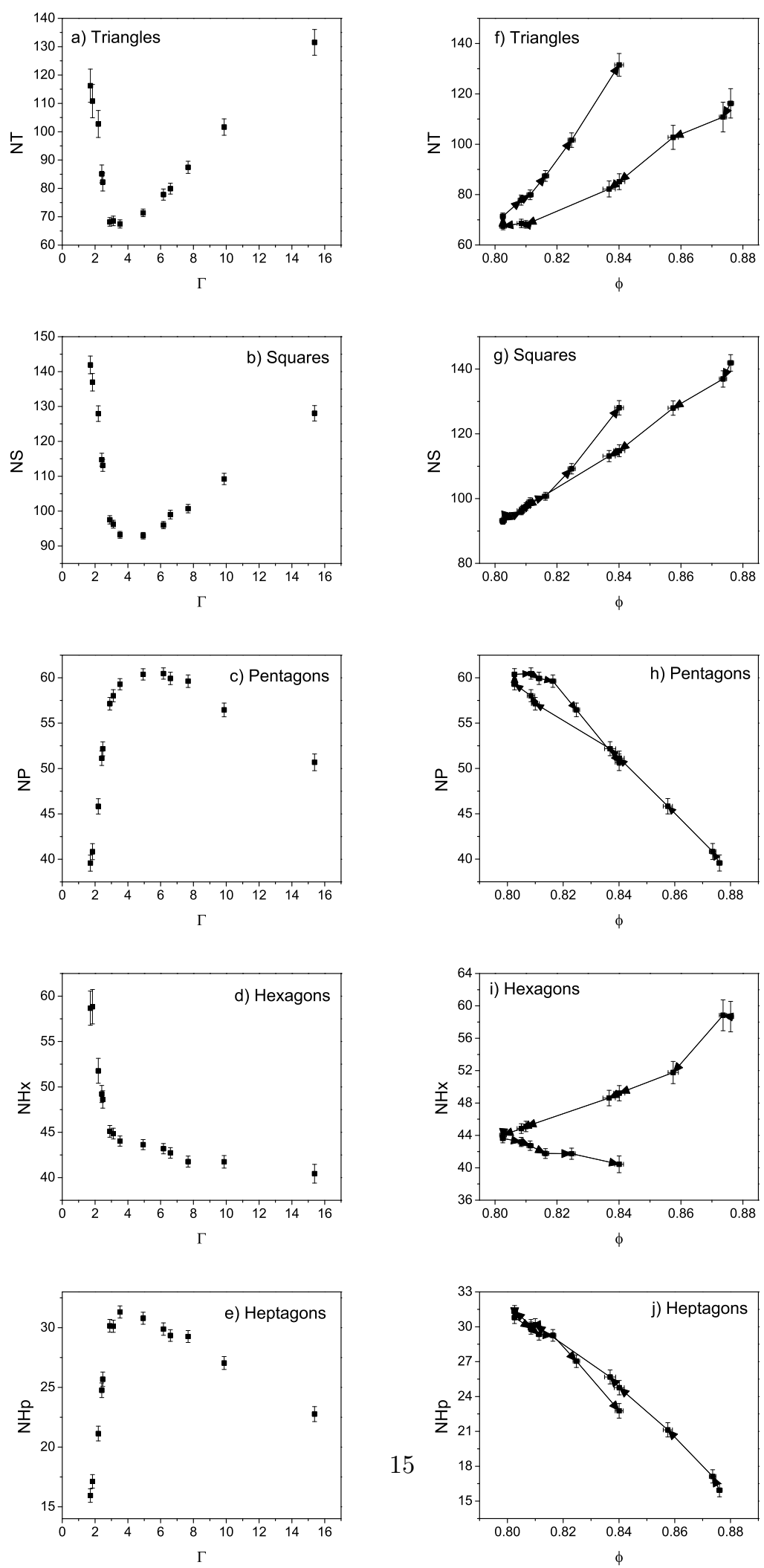

Figure 4: The populations of polygons defined in the text as a function of the tap intensity (a)-(e), and as a function of the mean packing fraction (f)-(j). The arrows indicate the direction of increasing $\Gamma$. 

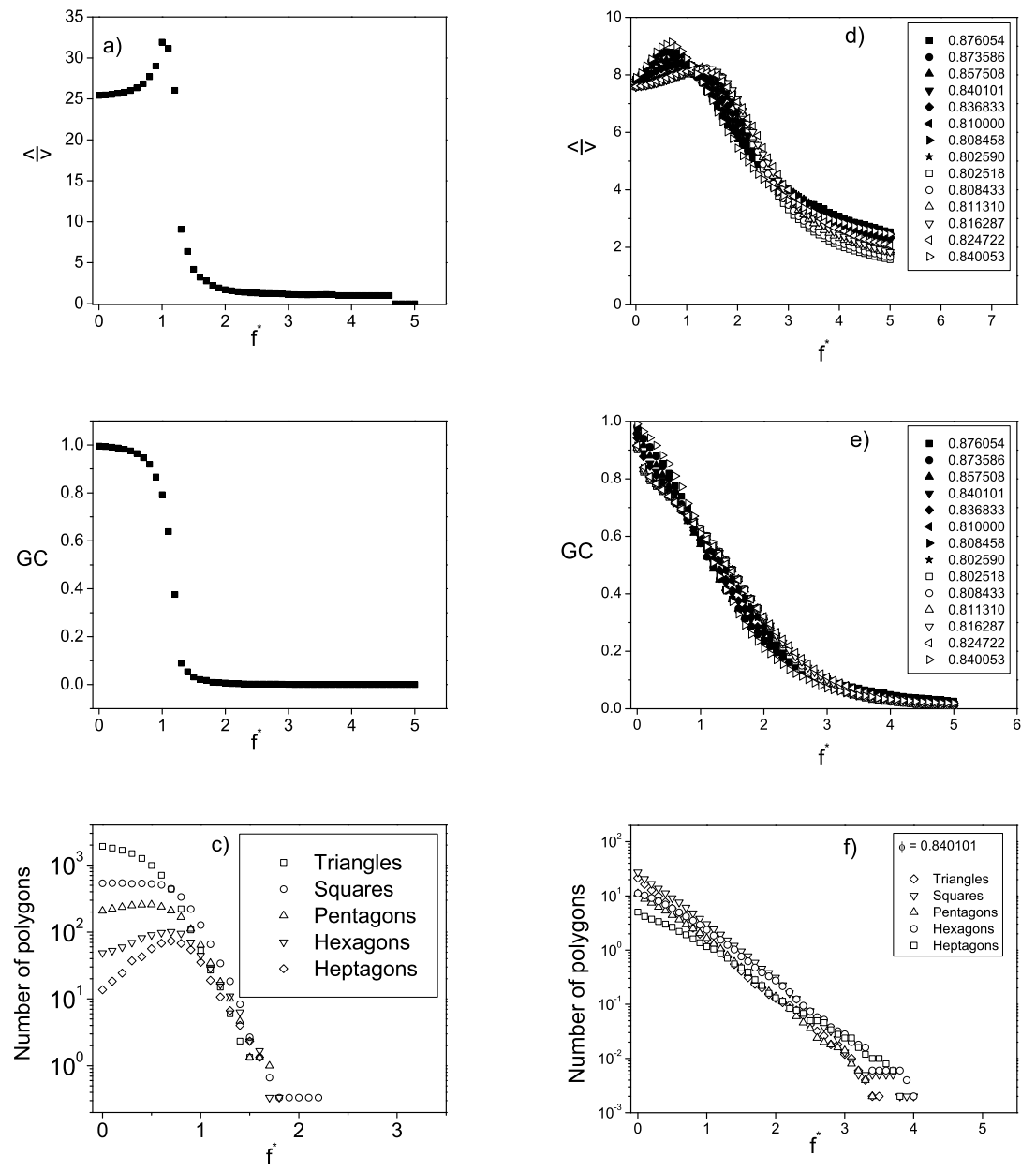

Figure 5: Average topological distance $\langle l\rangle$, giant component $C G$ and populations of polygons for compressed, jammed networks at $\phi=0.85$ (left) and tapped networks (right). 

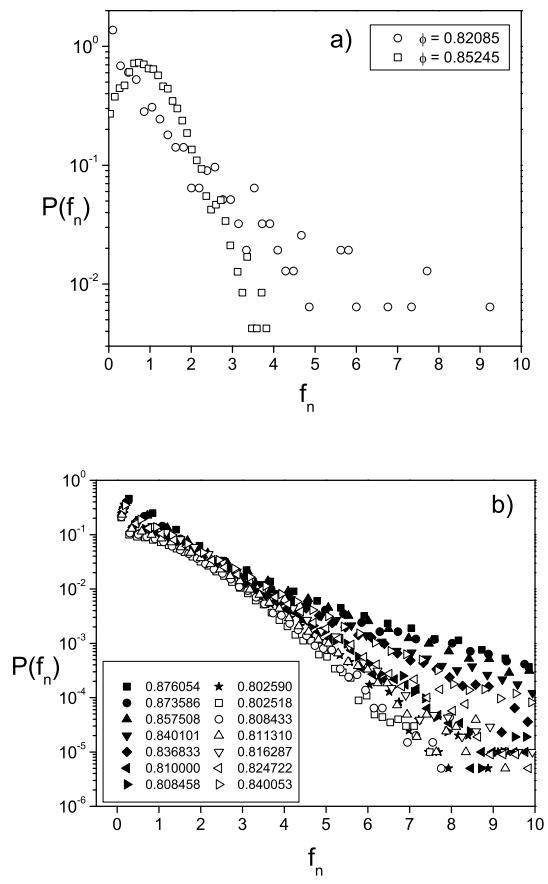

Figure 6: Normalized histogram of the normal contact forces for all the contacts. The variable $f_{n}=F_{n} /\left\langle F_{n}\right\rangle$ is the normal contact force normalized by the average. a) Results for a compressed system before $(\phi=0.82)$ and after jamming $(\phi=0.85)$. b) Results for the packing states reached by tapping. Note that the values of packing fraction are shown in the caption in order of increasing $\Gamma$. 


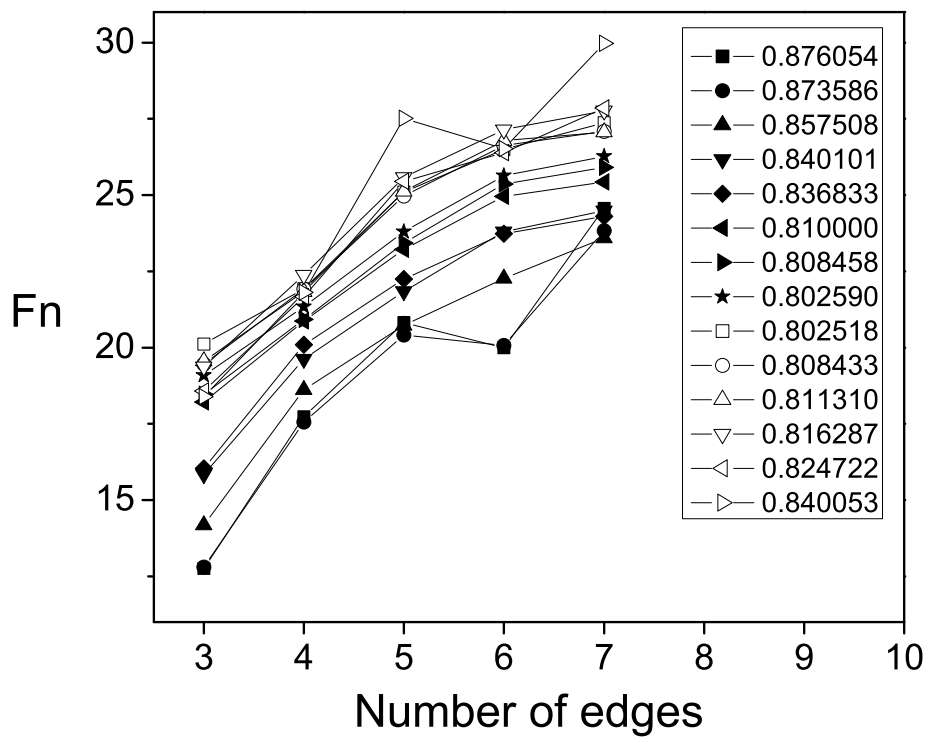

Figure 7: Normal force averaged over all the contacts of the polygons versus the size of the polygons. As in previous figures, the values of packing fraction are shown in the caption in order of increasing $\Gamma$. 